\title{
Redes de Monitoramento Ambiental: Um projeto aplicado às Geleiras Patagônicas
}

\author{
Guilherme T. Netto ${ }^{1}$, Jorge Arigony-Neto ${ }^{1}$, Adenauer Correa Yamim ${ }^{2}$ \\ ${ }^{1}$ Laboratório de Monitoramento da Criosfera - Universidade Federal do Rio Grande (FURG) \\ Rio Grande - RS - Brazil \\ ${ }^{2}$ Laboratório de Computação Ubíqua e Pervasiva - Universidade Federal de Pelotas (UFPEL) \\ Pelotas - RS - Brasil \\ guilherme.netto@inf.ufpel.edu.br, jorgearigony@furg.br, adenauer@inf.ufpel.edu.br
}

\begin{abstract}
The climate changes are an issue constantly debate today, and glaciers play an important role in this context as they are as natural sensors that react to these changes. Its monitoring is of great importance in order to know their behavior related to weather events and their influence on climate. This paper proposes the use of the sensor network model to carry out the monitoring of glaciers in an integrated manner, using a wide range of sensors. The first tests with automatic weather stations (AWS) were very promising even with severe weather conditions.
\end{abstract}

Resumo. As mudanças climáticas são um tema em constante debate atualmente, e as Geleiras têm um papel importante neste contexto, pois são como sensores naturais que reagem a estas mudanças. Seu monitoramento é de grande importância a fim de conhecermos seu comportamento perante a eventos climáticos e sua influência no clima. O presente trabalho propõe a utilização do modelo de redes de sensores para realizar o monitoramento de Geleiras de forma integrada, utilizando um conjunto variado de sensores. Os primeiros testes com estações meteorológicas automáticas (AWS) mostram-se promissores mesmo com condições climáticas severas.

\section{Introdução}

Nas últimas décadas a discussão sobre a influência humana nas mudanças climáticas tem se intensificado. Programas de âmbito mundial para tratar do tema tem se formado, como exemplo concreto neste sentido, temos o Painel Intergovernamental sobre Mudanças Climáticas (IPCC). Órgão criado pelo Programa das Nações Unidas para o Meio Ambiente (PNUMA) e pela Organização Meteorológica Mundial (OMM) em 1988 para estudar o problema das mudanças climáticas. Reúne 2.500 cientistas de mais de 130 países. O IPCC tem emitido relatórios periódicos caracterizando as influências dos sistemas humanos sobre os ecossistemas naturais. O último relatório publicado evidencia a ação humana como grande responsável pelo aquecimento global detectado nos últimos 50 anos. Este aquecimento atinge vários aspectos climáticos, dentre os quais destacase a elevação da temperatura dos oceanos, variações extremas de temperatura do ar e os padrões de circulação atmosférica [IPCC 2014].

Outro aspecto tratado por este relatório é a influência destas variações climáticas no derretimento das massas de gelo do planeta, o que traria consequências negativas de 
grandes proporções, como por exemplo o aumento do nível médio dos oceanos, alterações climáticas mais severas e a decorrente necessidade de migração populacional.

Estas massas de gelo que cobrem parte da superfície da terra, de forma perene ou sazonal, fazem parte da criosfera e cobrem $10 \%$ do total do planeta [CUFFEY and PATERSON 2010]. Segundo [Arigony-Neto 2006] houve uma grande aceleração no derretimento de parte destas massas, bem como desagregação de plataformas de gelo e a extinção de geleiras. Tais fenômenos podem influenciar o clima do planeta dada a importância da criosfera e do oceano austral nos processos de equilíbrio térmico do planeta [Mata et al. 2011, Simões 2014].

Geleiras são estruturas dinâmicas resultantes da ação do clima e da topografia. Formam-se por precipitação de neve, gelo ou chuva e perdem massa por derretimento e evaporação de neve, desprendimento de massas de gelo, erosão eólica e avalanches. Isto determina duas grandes áreas conceituais, a Área de acumulação e a Área de ablação [CUFFEY and PATERSON 2010]. O balanço entre acúmulo e perda de gelo determina a formação ou extinção de uma geleira.

Muitos esforços vem sendo empregados para monitorar o derretimento das geleiras [LaChapelle 1959, Müller and Keeler 1969, Bøggild et al. 2004, Braithwaite 2009, Hulth 2010, Van As 2011, Fausto et al. 2012] e diferentes estratégias com sensores específicos foram empregadas neste intuito, como a observação da variação das camadas de gelo por estacas [LaChapelle 1959, Müller and Keeler 1969], sensores de pressão [Bøggild et al. 2004, Van As 2011, Fausto et al. 2012], ultrassônicos [Hulth 2010, Van As 2011] e por fios de aço [Hulth 2010]. Cada estratégia mostrou pontos fortes e deficiências, demonstrando assim a necessidade de criarem-se sistemas híbridos, com mais de uma abordagem de aquisição dos dados e de forma distribuída.

Os parâmetros meteorológicos como vento (direção e intensidade), pressão atmosférica, temperatura do ar e do gelo, umidade e luminosidade são medidos por estações meteorológicas, que devido ao alto custo e à baixa portabilidade, são instaladas em número reduzido, limitando-se a poucas unidades. Esta resolução espacial não representa o que acontece em toda extensão das geleiras. Para monitorar o derretimento destas geleiras com uma maior precisão se faz necessária a utilização de mais de uma técnica de levantamento de dados, com uma maior resolução temporal para uma melhor calibração e validação nos estudos destes modelos de degelo, sobretudo em escalas de tempo menores em eventos específicos [Hulth 2010].

O emprego de mais de um ponto de coleta conectado a uma rede de sensores distribuídos pode contribuir significativamente para uma visão mais ampla destas dinâmicas (acúmulo e ablação). Desta maneira, comparando as informações a respeito da geleira, como temperatura e perda de massa, por exemplo, com dados meteorológicos, é possível verificar como os processos meteorológicos influenciam na perda de massa de gelo, sobretudo nos eventos extremos, não só nas zonas de ablação mas também nas zonas de acumulação, bem como em altitudes diversas e outras condições morfológicas.

A coleta automatizada de informações do meio por dispositivos computacionais, móveis ou não, com o intuito de qualificar o atendimento às necessidades dos usuários é chamada de computação ciente do contexto [Lopes et al. 2014].

Ampliando os conceitos de ciência do contexto, [Ye et al. 2012] propõe um mo- 


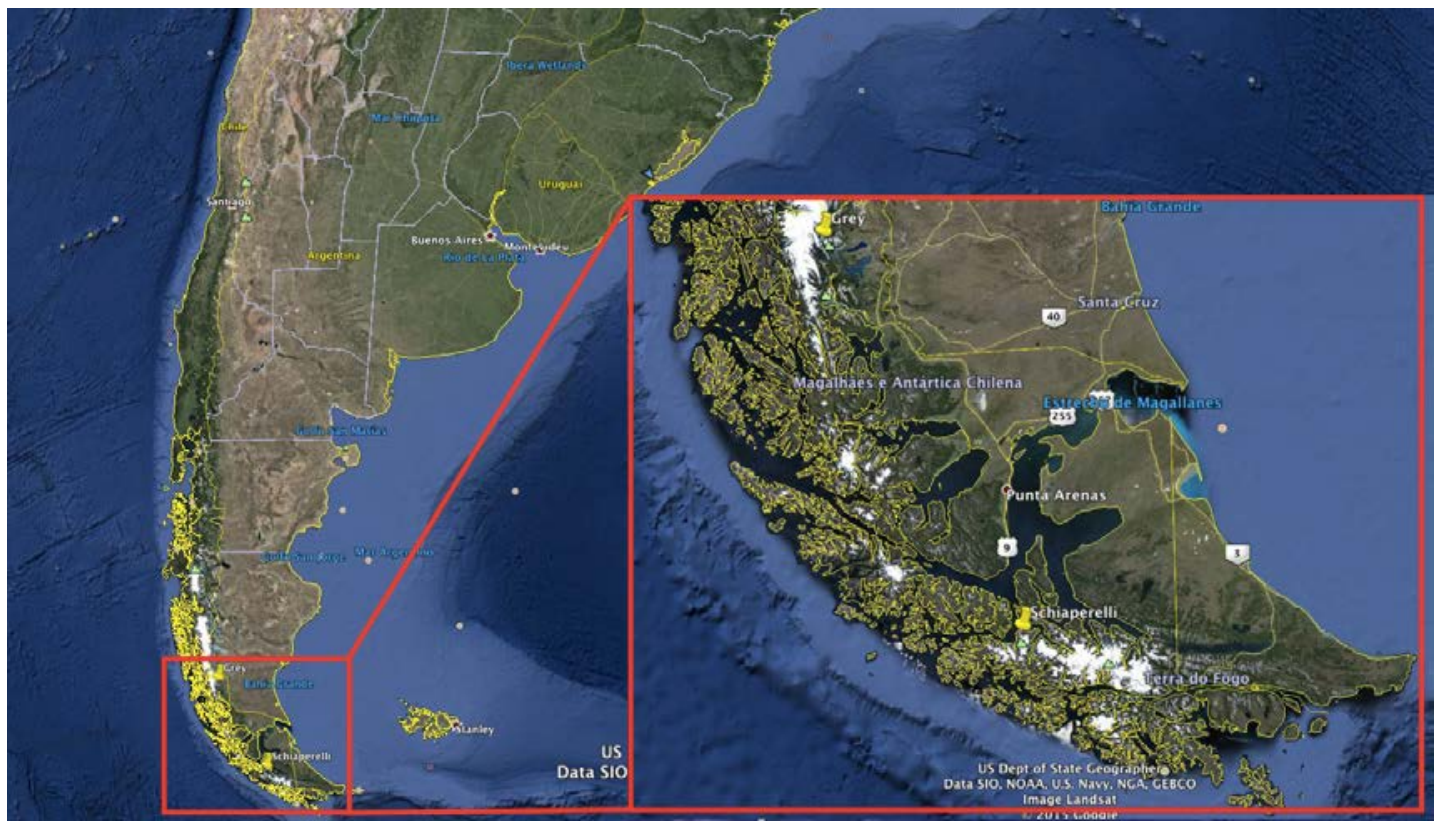

Figura 1. Mapa da Região de Estudo

delo de integração onde além da análise dos dados coletados do ambiente também é feito um acompanhamento da evolução dos mesmos, considerando mais uma dimensão, a análise temporal, introduzindo então o conceito de ciência de situação.

Neste sentido, este trabalho apresenta uma proposta para criação de uma rede de sensores meteorológicos e de ablação com o objetivo de monitorar um ambiente climático singular. As áreas de estudo situam-se ao sul da Patagônia Chilena, na Cordilheira Darwin, um pouco mais ao norte no Parque Nacional Torres del Paine, e chamam-se Geleira Schiaparelli e Geleira Grey (Vide Figura 1). As características geográficas, o isolamento e a baixa influência direta de fatores antrópicos tornam esta região propícia para este tipo de estudo.

\section{Metodologia}

A miniaturização de alguns sensores sem fio disponíveis atualmente e a distribuição integrada dos mesmos junto ao meio físico são indicativos de que as Redes de Sensores sem Fio são uma importante contribuição para consolidação da Computação Ubíqua.

Com os recentes avanços no desenvolvimento de sistemas microeletrônicos, as Redes de Sensores sem Fio podem ser formadas por um grande número de pequenos sensores, onde cada sensor é um elemento autônomo capaz de captar a informação do meio, tratar e enviar esta informação através de comunicação sem fio, consolidando assim a autonomia prevista na Computação Ubíqua [Lopes et al. 2014].

Para tanto, se faz necessário um transceptor para comunicação, uma unidade de sensoriamento, fonte de energia, memória e uma unidade de processamento. Tais elementos influenciaram várias aplicações desta tecnologia que agrega recursos antes intratáveis com modelos convencionais, por exemplo, no monitoramento de áreas remotas, com sensores sendo lançados sobre reservas ambientais, florestas, vulcões, rios [Ruiz 2003]. 
Por esse motivo, o presente trabalho busca agregar um conjunto de tecnologias, preferencialmente livres (i.e., projeto de domínio público), de baixo custo, mas perseguindo um nível de confiabilidade aceitável para medidas da dinâmica de geleiras. O desenvolvimento da rede de sensores está sendo feito de maneira incremental, pois existe a necessidade da validação dos sensores de borda para posteriormente interligá-los utilizando uma rede.

Na primeira etapa foram desenvolvidos dois conjuntos de sensores que ainda funcionam de maneira isolada, uma Estação Meteorológica Automática (AWS) e um sensor de Ablação utilizando tags RFID. Na segunda etapa estes sensores serão interligados utilizando uma rede sem fio e comunicação satelital com o servidor remoto responsável pelo registro e processamento das informações coletadas.

\subsection{Ambiente Operacional}

$\mathrm{Na}$ implementação da rede de sensores estão sendo utilizados os microcontroladores da família Arduino [Arduino 2016], que possuem as características técnicas necessárias ao projeto, e destacam-se pelo seu baixo custo e praticidade de desenvolvimento.

Para a comunicação de dados na rede projeta-se a utilização das placas ESP8266 versão 7, que possibilitam a conexão entre os nodos e o concentrador via protocolo TCP e possuem interface para antenas externas possibilitando um ganho de sinal maior comparado às outras versões. Já a comunicação remota da rede, concentrador e servidor deverá ser feita utilizando a tecnologia de Rádio-Pacote através de uma rede satelital de Rádio Amadores. Este modelo apresenta uma banda de comunicação reduzida, mas suficiente para as demandas de comunicação da proposta de monitoramento que está sendo proposta. O modelo de sensores de borda é composto por conjuntos de circuitos acoplados

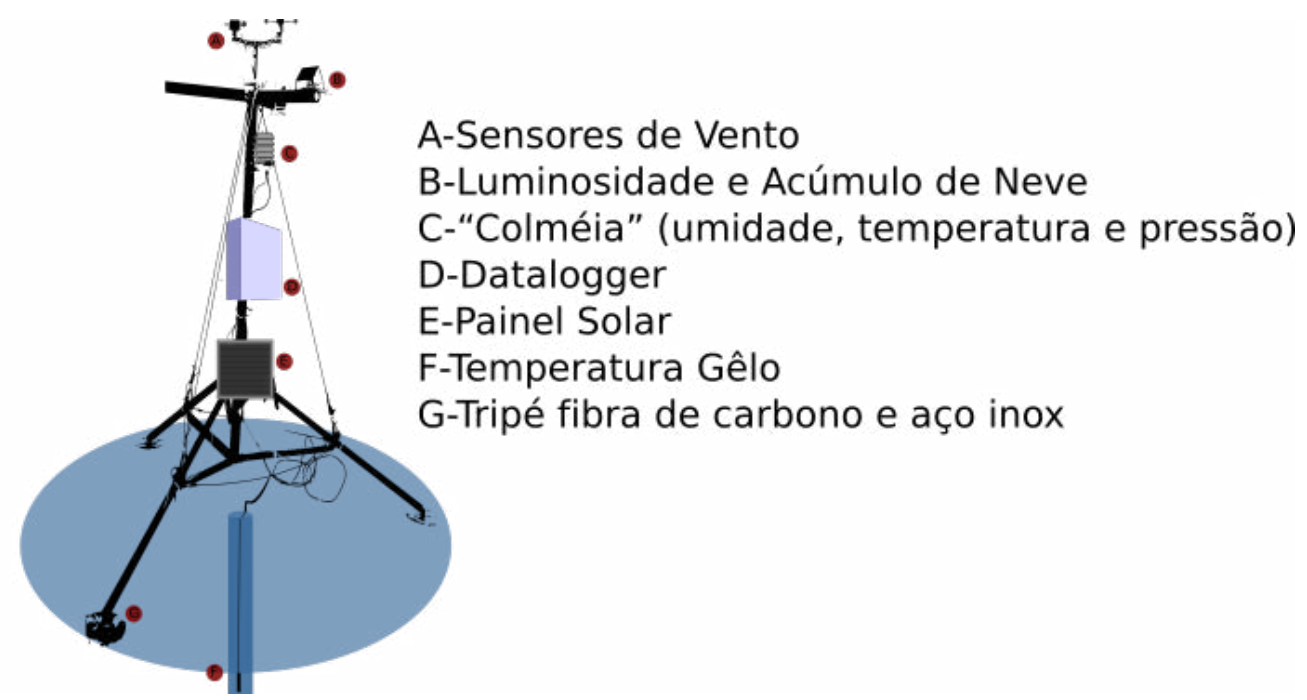

Figura 2. Estação Meteorológica Automática

pelos microcontroladores através de comunicação sem fio. Cada conjunto deverá possuir sensores para medir velocidade de fluxo das geleiras, pressão barométrica, temperatura do ar, umidade, temperatura da neve, quantidade de neve acumulada, ablação, radiação solar, direção e intensidade de vento, como mostra a Figura 2.

Um protótipo de estação meteorológica completa foi executado com tripé em fibra 
de carbono, a um custo de $\mathrm{R} \$ 2.000,00$, bastante inferior ao custo das estações comerciais aquisitadas anteriormente com características semelhantes, e que têm um preço médio de $\mathrm{R} \$ 40.000,00$. Pelo fato da estação ser construída com materiais leves o seu peso total é $11 \mathrm{Kg}$, mostrando-se bastante portátil quando comparada com as estações anteriormente instaladas que pesam $40 \mathrm{Kg}$ aproximadamente.

Os sensores de ablação usualmente são desenvolvidos a partir do modelo de balizas, o qual consiste em inserir um conjunto de canos de PVC na geleira até 12 metros de profundidade, e a cada retorno da equipe ao campo são efetuadas medições de quanto estas balizas estão aparentes, apresentando assim uma baixa resolução temporal, em torno de seis meses.

No modelo proposto a detecção do descongelamento superficial é feito por sensores de reconhecimento por radiofrequência(RFID), Figura 3, com uma resolução espacial de $15 \mathrm{~cm}$ e um datalogger, assim será possível armazenar os dados de derretimento das geleiras nas regiões de ablação nos períodos exatos que ocorrem.
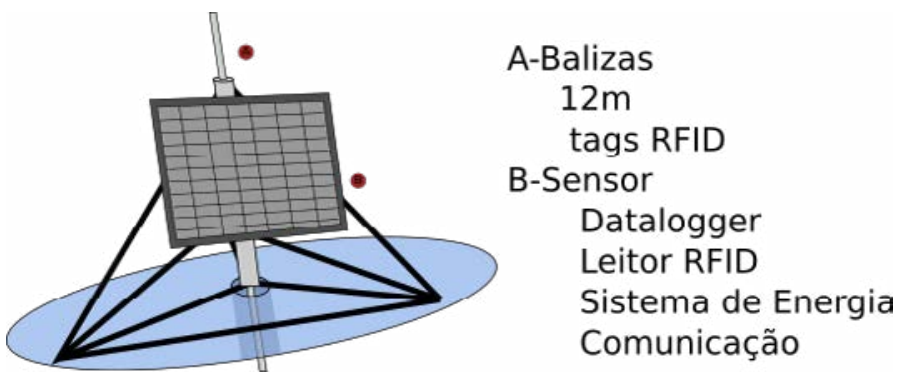

Figura 3. Sensor de Ablação

O modelo de integração será implementado utilizando-se uma arquitetura em camadas, com sensores de borda realizando a coleta das informações, uma camada intermediária chamada de middleware, responsável pela distribuição e transmissão das informações e os servidores de contexto que serão os responsáveis pelo armazenamento temporário e transmissão para a base remota. Tal abordagem torna o projeto bastante versátil quanto à customização da rede de sensores como demonstrou o trabalho de [Ruiz 2003] e [Neto 2009].

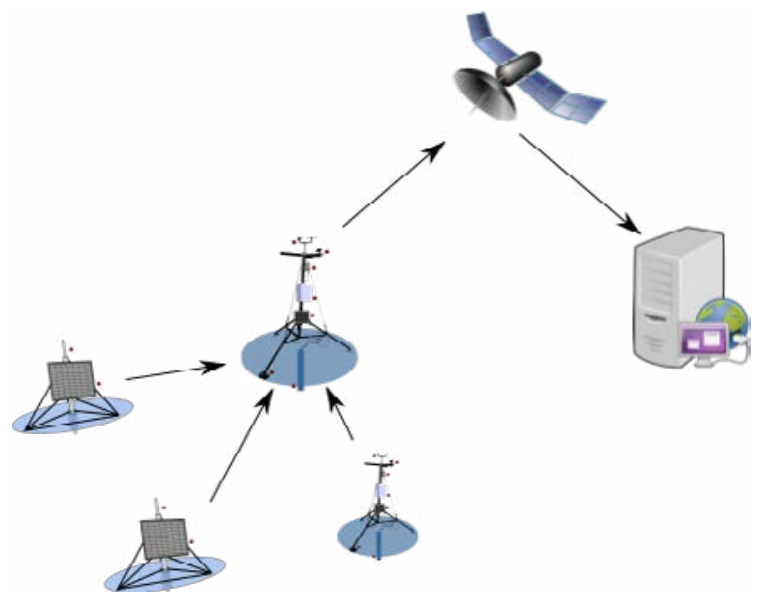

Figura 4. Topologia da rede 
A topologia de rede a ser utilizada está ainda sob estudo, e o modelo mais adequado até este momento do estudo é o multihop, devido a possibilidade de cobrir distâncias maiores entre os nodos sensores e o concentrador.

\section{Testes de Campo}

Os primeiros testes de campo foram realizados com as Estações Meteorológicas Automáticas, e o objetivo destes experimentos era testar a robustez dos conjuntos. Testes de bancada foram efetuados durante o mês de julho de 2015 e as duas estações foram instaladas junto às estações meteorológicas da Furg, Figura 5, Campus Carreiros, para fins de aferição. Esta é uma típica linha de registro com dados gravados pela AWS:

$25.08 .15 ; 10: 23: 22 ; 491.00 ; 5.00 ; 19.69 ; 35.50 ; E ; 62.40 ; 18.70 ; 18.40 ; 1019$

Nesta linha os dados são separados por (;) e representam respectivamente, data e hora da leitura, luminosidade, acúmulo de neve, temperatura do gelo, velocidade do vento, direção do vento, umidade relativa do ar, temperatura do ar em dois sensores e pressão atmosférica. A frequência de leitura é de um registro por minuto.

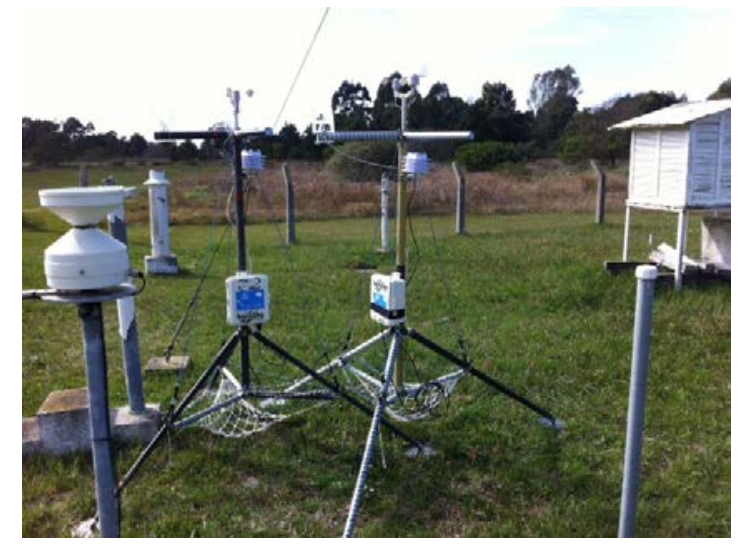

Figura 5. Teste de Campo - Furg/Campus Carreiros

Com a experiência dos testes de bancada, ajustes foram feitos para adequar os equipamentos para o ambiente de estudo e em setembro de 2015 as duas AWS foram instaladas nas Geleiras Patagônicas. Em conjunto com o Instituto Antártico Chileno, Fundação CEQUA e Universidade de Magalhães, todos com sede em Punta Arenas no Chile, foram realizadas duas expedições, a primeira para instalação da estação na Geleira Schiaparelli(Figura 6), Cordilheira Darwin, e a segunda para instalação na Geleira Grey(Figura 7), Parque Nacional Torres del Paine. A Estação de Apoio Antártico(ESANTAR) também deu apoio fornecendo parte dos equipamentos de gelo.

Instalar e testar equipamentos em campo via de regra são tarefas bastante árduas, sobre tudo se as áreas de estudo estão em regiões remotas. É o caso das geleiras estudadas, principalmente a Schiaparelli, que exigiu uma logística bastante elaborada devido a localização geográfica, pela exposição a condições severas de clima(baixas temperaturas, ventos fortes e neve) e possuir um terreno bastante acidentado.

Para exemplificar, na primeira expedição houve um deslocamento de barco desde Punta Arenas, Chile, atravessando o Estreito de Magalhães, onde após o desembarque na praia a equipe precisou realizar o porteio dos equipamentos por dois dias até o campo 
base, e somente então conseguir o acesso a geleira e começar os trabalhos no gelo propriamente dito. Isto exigiu alguns conhecimentos e treinamento específicos de escalada em gelo e deslocamento em geleiras, bem como uma preparação física prévia.
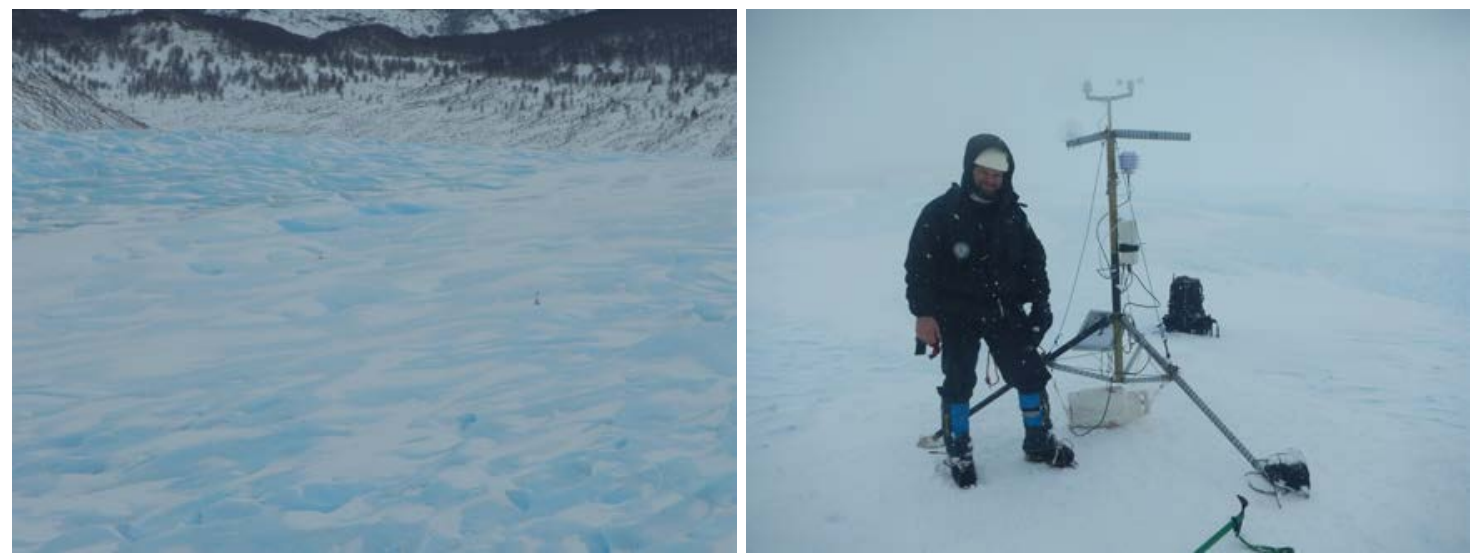

Figura 6. Geleira Schiaparelli (54 24'1.50"S 70 52'39.93"O) e AWS instalada

A escolha da Geleira Schiaparelli ocorreu por ser uma região bastante inóspita, com baixa influência antropogênica direta e por estar fortemente influenciada pelas forçantes climáticas provenientes da Antártida (vento, precipitação e umidade). A escolha também se deu por conveniência logística visto que a Os institutos chilenos citados anteriormente frequentemente realizam expedições nesta região, o que facilita enormemente as operações de instalação e manutenção.
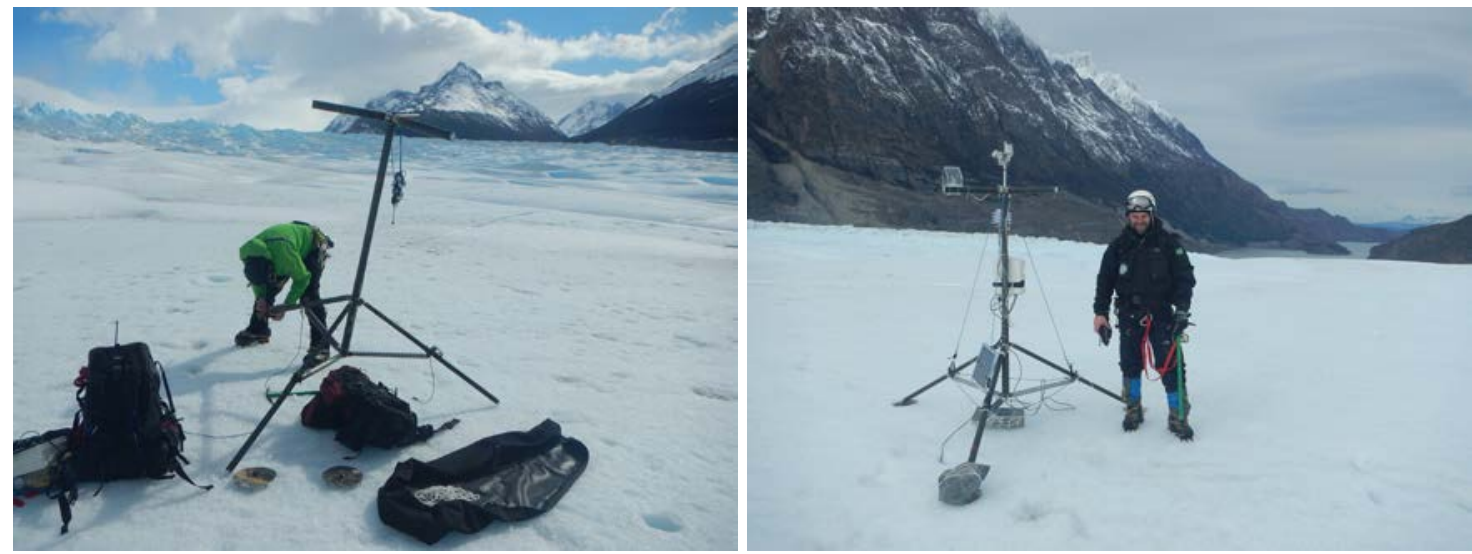

Figura 7. Geleira Grey (54 24'1.50"S 70 52'39.93"O) e Montagem da AWS

A Geleira Grey também foi escolhida pela conveniência do apoio dos institutos chilenos, e pelo fato de ser um ambiente diverso, com um clima mais seco e apresentar dimensões maiores e um período de estudos pretéritos mais longo.

\subsection{Primeiros Resultados}

As duas estações estão em operação desde setembro de 2015, e em janeiro de 2016 foi trocado o cartão de memória da AWS Grey. Foi verificado seu funcionamento e condições físicas gerais. O sistema está operando de maneira satisfatória, tendo armazenado 172.625 leituras. Foi verificado apenas um incidente com um conjunto de sensores devido a uma falha em um conector que foi danificado devido ao congelamento. A estrutura 


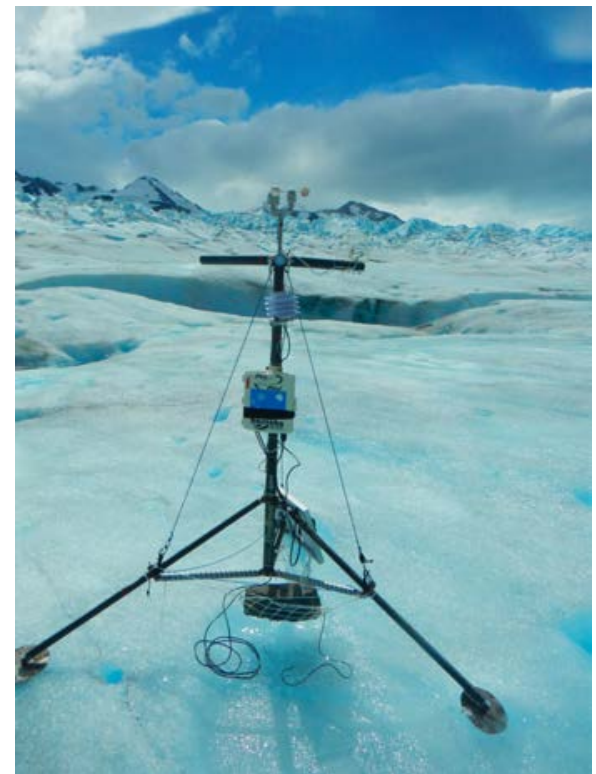

Figura 8. AWS Grey após 6 meses de funcionamento

física mostrou-se extremamente robusta, apesar do baixo peso. A Figura 8 mostra a AWS Grey após 6 meses de operação, demonstrando que as estratégias de ancoragem e escolha de materiais utilizados foram plenamente satisfatórias. No mês de abril será efetuada a troca do cartão de memória da AWS Schiaparelli.

\section{Conclusões}

Este trabalho tem como principal contribuição o desenvolvimento de um conjunto de tecnologias para o monitoramento de geleiras e o estudo de seu comportamento.

Os primeiros testes mostraram que a abordagem utilizada, aplicando plataformas livres e de baixo custo, foi bastante satisfatória. Com esta experiência será possível implantar o modelo de redes na próxima temporada, em setembro de 2016. Nesta nova etapa, já com os inéditos sensores de ablação com leitores RFID, será feito um acompanhamento com uma resolução temporal bem superior à utilizada atualmente.

É esperado que com este conjunto de dados, tendo uma resolução espacial e temporal maior que as utilizadas até então, seja possível correlacionar o comportamento destas geleiras com eventos climáticos, estimar de forma mais precisa as velocidades de deslocamento e seu cálculo de balanço de massa de gelo.

\section{Referências}

Arduino (2016). Arduino Web Page. https : / / www . arduino . cc/. Accessed: 2016$01-15$.

Arigony-Neto, J. (2006). Monitoring glacier parameters on the Antarctic Peninsula - a centerline approach combining satellite and GIS data. $\mathrm{PhD}$ thesis.

Bøggild, C. E., Olesen, O. B., Ahlstrøm, A. P., and Jørgensen, P. (2004). Automatic glacier ablation measurements using pressure transducers. Journal of Glaciology, 50(169):303-304. 
Braithwaite, R. J. (2009). Calculation of sensible-heat flux over a melting ice surface using simple climate data and daily measurements of ablation. Annals of Glaciology, 50(50):9-15.

CUFFEY, K. and PATERSON, W. S. B. (2010). The Physics of Glaciers.

Fausto, R. S., van As, D., Ahlstrom, A. P., Andersen, S. B., Andersen, M. L., Citterio, M., Edelvang, K., Larsen, S. H., Machguth, H., Nielsen, S., and Weidick, A. (2012). Ablation observations for 2008-2011 from the Programme for Monitoring of the Greenland Ice Sheet (PROMICE). Geological Survey of Denmark and Greenland Bulletin, (26):73-76.

Hulth, J. (2010). Using a draw-wire sensor to continuously monitor glacier melt. Journal Of Glaciology, 56(199):922-924.

IPCC (2014). Climate Change 2014: Synthesis Report. Contribution of Working Groups I, II and III to the Fifth Assessment Report of the Intergovernmental Panel on Climate Change. Technical report.

LaChapelle, E. (1959). ERRORS IN ABLATION MEASUREMENTS. Journal of Glaciology, 3(26).

Lopes, J., Souza, R., Geyer, C., Costa, C., Barbosa, J., Pernas, A., and Yamin, A. (2014). A middleware architecture for dynamic adaptation in ubiquitous computing. Journal of Universal Computer Science, 20(9):1327-1351.

Mata, M. M., José Goldemberg and Jefferson Cardia Simões and Heitor Evangelista and Lúcia de Siqueira Campos and Carlos Alberto Eiras Garcia, and Breme, U. F. (2011). Antártica e as Mudanças Globais: um desafio para humanidade. São Paulo, 1 edition.

Müller, F. and Keeler, C. M. (1969). Errors in short-term ablation measurements on melting ice surfaces. Journal of Glaciology, 8(52):91-105.

Neto, A. T. (2009). Rede de sensores sem fio e computação ubíqua na agropecuária. Embrapa, page 19.

Ruiz, L. B. (2003). Maná: uma arquitetura para gerenciamento de redes de sensores sem fio. $\mathrm{PhD}$ thesis.

Simões, J. C. (2014). Antartica, 2048: Mudanças Climáticas e Equilíbrio Global. pages $50-59$.

Van As, D. (2011). Warming, glacier melt and surface energy budget from weather station observations in the melville bay region of northwest greenland. Journal of Glaciology, 57(202):208-220.

Ye, J., Dobson, S., and McKeever, S. (2012). Situation identification techniques in pervasive computing: A review. Pervasive and Mobile Computing, 8(1):36-66. 